\title{
Search for extended sources of neutrino emission with 7 years of IceCube data
}

\author{
The IceCube Collaboration ${ }^{\dagger}$ \\ $\dagger$ http://icecube.wisc.edu/collaboration/authors/icrc17_icecube \\ E-mail: epinateicecube.wisc.edu, juaguila@ulb.ac.be
}

The IceCube Neutrino Observatory has demonstrated the existence of an astrophysical highenergy neutrino flux with a significance exceeding $7 \sigma$. Nevertheless, the observed astrophysical neutrino flux shows no clear association with any known source class so far. The most recent searches for point sources using seven years of data from IceCube have found no significant clustering of neutrino events nor correlation with preselected sources. However not all potential neutrino sources would appear point-like, and it is therefore important to widen the search to different source topologies. The high-energy gamma-ray and cosmic-ray skies show clear indications of extended structures. In the case of cosmic-ray interactions in these environments, both gamma-ray and neutrino emission are expected, and the detection of this neutrino flux would represent unambiguous proof of the hadronic nature of the sources. In addition, an extended source search could improve sensitivity to several point sources located close together in space, for example the Cygnus region, even if the individual fluxes are too low to be detected separately. Here we present the results of an extended source analysis with seven years of IceCube data. Because the extensions of potential sources are not always known a priori, five different extensions have been considered, from $1^{\circ}$ to $5^{\circ}$. All the five maps are consistent with the background-only hypothesis.

Corresponding authors: Elisa Pinat and Juan Antonio Aguilar*

Université Libre de Bruxelles, Science Faculty CP230, B-1050 Brussels, Belgium

35th International Cosmic Ray Conference - ICRC2017

10-20 July, 2017

Bexco, Busan, Korea

* Speaker. 


\section{Introduction}

The IceCube Neutrino Observatory is a neutrino detector located at the South Pole, encompassing a cubic kilometer of Antarctic ice between depths of $1450 \mathrm{~m}$ and $2450 \mathrm{~m}$ [1]. Completed in 2011, it is the largest neutrino detector built to date. During the construction period data was collected in four different string configurations, until the final 86 string configuration was reached. Similarly to other neutrino detectors, IceCube exploits the fact that charged particles, resulting from neutrino interactions, move faster than the phase velocity of light in the ice and emit Cherenkov radiation.

Neutrinos are ideal messengers, and their use in astronomy was imagined already in the 1960s. Stars, Active Galactic Nuclei (AGNs) and astrophysical engines are opaque to photons, while neutrinos can easily escape, preserving directional information and carrying information from the densest regions of the Universe. Astrophysical neutrinos are also tracers of hadronic interactions, and the identification of their sources may help to clarify cosmic ray acceleration processes.

The detection of a diffuse high-energy astrophysical neutrino flux made in 2013 by the IceCube collaboration is now established at more than $7 \sigma$ confidence level with both events starting within the detector (all flavor) [2] and events traversing through the Earth $\left(v_{\mu}\right.$ charged-current) [3]. However, the sources of this neutrino flux have not been identified yet [4]. Finding neutrino point sources in the sky requires locating an excess of events from a particular direction over the background of atmospheric neutrinos and muons. Together with the spatial component, also the energy distribution of events is used as discriminator between the signal and the background hypotheses. Since point source searches have been unfruitful so far, it is important to widen the search to different source topologies to maximize the discovery potential. A different topology are extended sources that are motivated by clear indications of extended structures seen in the highenergy gamma-ray and cosmic-ray skies.

In these proceedings we present the result of the search of spatially extended sources with seven years of IceCube data from three years of operation in partial levels of completion (IC-40, IC-59 and IC-79) and the first four years of the completed 86 string detector (IC86-I, IC86-II, IC86-III and IC86-IV). Data have been collected from May 2008 to May 2015, for a total of 713752 events after event selection for through-going muon neutrinos (see [4]) in 2426.2 days of livetime. The previous extended source analysis was limited to four years, using samples from IC40 to IC86-I, and the results are shown in [5]. The likelihood method has been improved by accounting for signal contamination in the background estimation. With this new method, sensitivities and discovery potentials have been calculated, and five significance skymaps have been produced, testing extensions from $1^{\circ}$ to $5^{\circ}$.

\section{Physics case}

Motivations for searching for extended sources are manifold. Extended sources have been detected in the $\gamma$-sky. For example, the H.E.S.S. survey of the inner part of the Galactic Plane [6] revealed a number of bright extended $\gamma$-ray sources. If the observed $\gamma$-rays are produced by cosmic-ray interactions, a very-high-energy neutrino flux should be associated as well. If detected, this would represent an unambiguous proof of the hadronic nature of the sources. Despite many 


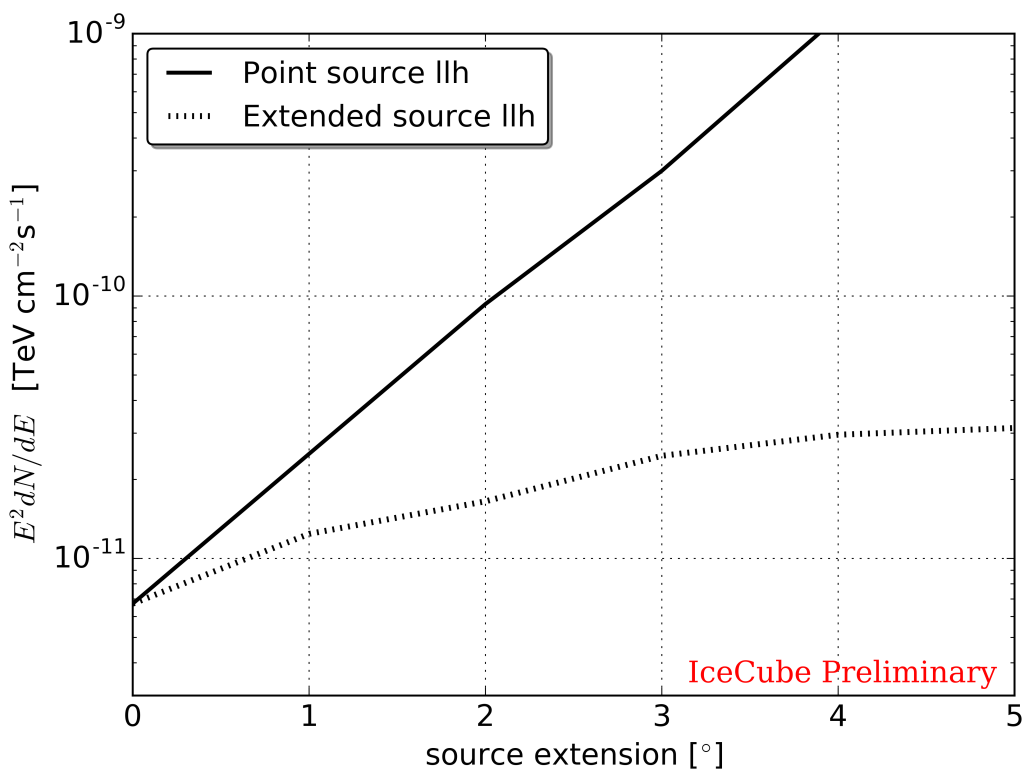

Figure 1: Discovery potential for an $\mathrm{E}^{-2}$ flux from an extended source at a declination of $16^{\circ}$ for one year of IC86 data with no trial factor correction. The regular point source analysis (solid line) is compared to the discovery potential of the extended source analysis (dotted line), where the latter employs the known source extension.

$\gamma$-ray sources show an extension of about $0.3^{\circ}-0.4^{\circ}$, which is below IceCube's median angular resolution of about $1^{\circ}$ at $1 \mathrm{TeV}$, some sources show even larger extension, thus providing a strong motivation to look for extended sources.

The high-energy cosmic-ray sky shows also clear indications for extended structures: altough being at larger scales and higher energies, the Telescope Array has observed a 5.1 $\sigma$ excess in cosmic rays with energies above $57 \mathrm{EeV}$ in a region centered on coordinates $146.7^{\circ} \mathrm{RA}, 43.2^{\circ} \mathrm{DEC}$ [7], hotspot larger than the extension expected by propagation effects. In addition, the Kascade Grande experiment has observed at $5.5 \sigma$ significance a $1^{\circ}$ extended $300 \mathrm{TeV}$ cosmic-ray hotspot in a mainly isotropic sky [8].

Finally, motivation in favor of a dedicated extended source analysis can also be found in Figure 1. Assuming the presence of extended sources and modeling the signal injection according to their extension, this plot shows the discovery potential for an $\mathrm{E}^{-2}$ flux of the regular point source analysis method that relies on an unbinned likelihood maximization, compared to the one calculated with our extended source analysis, with the spatial extension properly accounted for. In this case, the same value used to simulate the spatial extension enters the likelihood calculation, showing that, when the correct source extension is used, the improvement can be substantial. A point source analysis risks to be blind in the presence of an hypothetical extended source.

\section{Analysis Method}

To calculate the significance of a possible excess of neutrino events for a given direction in the sky over the background of atmospheric muons and neutrinos, this analysis uses an unbinned 
maximum likelihood method similar to the one described in [9]. The method exploits the fact that not only signal events from a neutrino source are expected to cluster in space, but also their energy spectrum is different from those of the backgrounds. Signal events follow an harder spectrum than atmospheric muons and neutrinos [10], and it has been demonstrated that the introduction of the energy term performs better than the simple spatial approach [9]. The likelihood expression adopted in standard point source searches is defined as:

$$
L\left(n_{s}, \gamma\right)=\prod_{i=1}^{N}\left(\frac{n_{s}}{N} S\left(\vec{x}_{s}, \vec{x}_{i}, \sigma_{i}, E_{i} ; \gamma\right)+\left(1-\frac{n_{s}}{N}\right) B\left(\delta_{i}, E_{i}\right)\right),
$$

where $S$ and $B$ are the signal and background probability density functions respectively, $n_{s}$ is the unknown number of signal events for a flux following a spectral index $\gamma$ and $N$ total number of events in the data sample under evaluation. The error associated to the reconstructed direction is represented by $\sigma_{i}, E_{i}$ is the reconstructed energy. Both the signal and background PDFs contain a spatial and an energy term, $S=S_{\text {space }} \times S_{\text {energy }}$ and $B=B_{\text {space }} \times B_{\text {energy }}$. The two parameters maximized in the likelihood are the number of signal events $n_{s}$ and the spectral index of the extended source assumed to be located at $\vec{x}_{s}$ where the likelihood is being evaluated.

The first modification with respect to the standard point source searches affects the term $S_{\text {space }}$. The signal spatial probability density function is assumed to be Gaussian but the point spread function, instead of depending only on the error on the reconstructed direction $\sigma_{i}$, is now convoluted with the source extension $\sigma_{s r c}$, resulting in:

$$
S_{\text {space }}=\frac{1}{2 \pi\left(\sigma_{i}^{2}+\sigma_{s r c}^{2}\right)} \exp \left(-\frac{\left|\vec{x}_{i}-\vec{x}_{s}\right|^{2}}{2\left(\sigma_{i}^{2}+\sigma_{s r c}^{2}\right)}\right)
$$

where $\sigma_{s r c}$ is fixed to one of the several discrete values from $1^{\circ}$ to $5^{\circ}$ and $\left|\vec{x}_{i}-\vec{x}_{s}\right|$ is the angular distance to the source.

The second modification is related to the estimate of the background using data, whose energy and spatial distribution are $B_{\text {space }}$ and $B_{\text {energy. }}$. The spatial background PDF $B_{\text {space }}$ depends only on declination, and is right ascension independent due to Earth's rotation. In a point source analysis, any hypothetical point source has a very small contribution in the declination band of interest, and is treated as negligible when building background PDFs using spatial and energy distribution of the data. In contrast, the contribution of an extended source within its declination band is not negligible anymore, and a small correction needs to be applied. To account for this contribution, a third PDF, called scrambled signal $\tilde{S}\left(\delta_{i}, E_{i} ; \gamma\right)$, is needed. The term is self explanatory, as the scrambled signal represents how a signal contribution looks like when scrambled in right ascension. The background derived from data is expressed in term of a mixture of this scrambled signal and the pure background $B\left(\delta_{i}, E_{i}\right)$ :

$$
\tilde{D}\left(\delta_{i}, E_{i}\right)=\frac{n_{s}}{N} \tilde{S}\left(\delta_{i}, E_{i} ; \gamma\right)+\left(1-\frac{n_{s}}{N}\right) B\left(\delta_{i}, E_{i}\right)
$$

The new signal-subtracted likelihood is finally expressed as:

$$
L\left(n_{s}, \gamma\right)=\prod_{i=1}^{N}\left(\frac{n_{s}}{N} S\left(x_{i}, \sigma_{i}, E_{i} ; \gamma\right)+\tilde{D}\left(\delta_{i}, E_{i}\right)-\frac{n_{s}}{N} \tilde{S}\left(\delta_{i}, E_{i} ; \gamma\right)\right)
$$




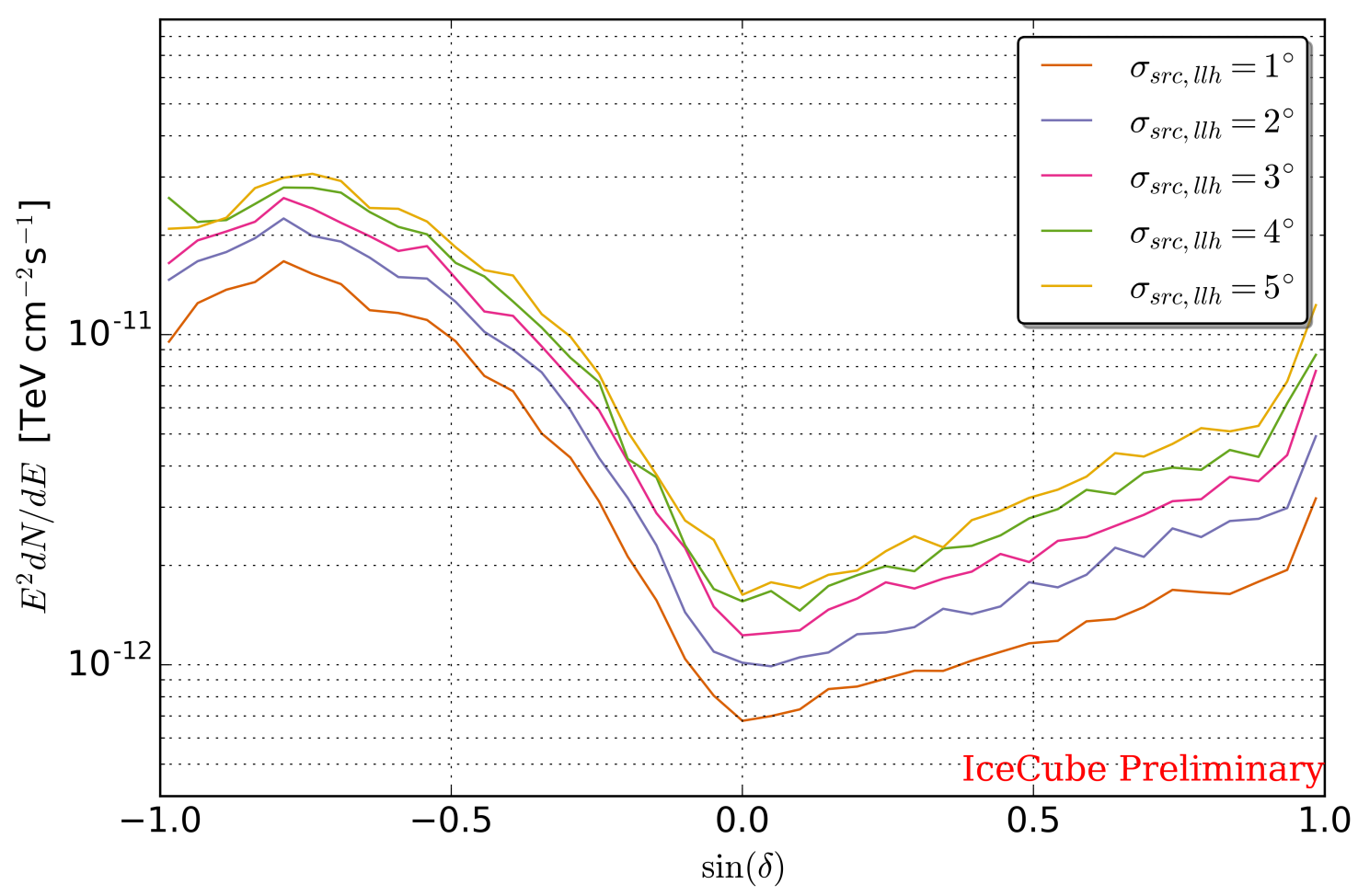

Figure 2: Sensitivity at $90 \%$ confidence level for the five extensions considered and an $E^{-2}$ power law spectrum. The best case scenario is assumed, that is when the simulated source extension $\left(\sigma_{s r c}\right)$ matches exactly the extension of the likelihood scan $\left(\sigma_{l l h}\right)$.

The scrambled signal PDF has an energy and a spatial term, $\tilde{S}=\tilde{S}_{\text {space }} \times \tilde{S}_{\text {energy. }}$. In this analysis, as in standard point-source ones, the signal energy term $S_{\text {energy }}$ is described by a power law, and the same power law is used to describe $\tilde{S}_{\text {energy }}$. The spatial part, $\tilde{S}_{\text {space }}$, depends on the description adopted for the signal spatial PDF $S_{\text {space. }}$. In our case the signal spatial PDF is modeled as 2D Gaussian, and therefore the scrambled signal spatial PDF is described as a 1D Gaussian with the sole dependence on the declination, divided by the cosine of the declination.

\section{Performance}

This analysis is sensitive to TeV-PeV neutrino sources in the Northern Hemisphere, and to PeV-EeV sources in the Southern Hemisphere, due to an energy constraint imposed by the need to reject the atmospheric muon background. The difference in the background rates for the Northern and Southern Hemispheres affects the shape of the two figures of merit of the analysis, the sensitivity and the discovery potential. The sensitivity, shown in Figure 2, represents the $90 \%$ confidence level upper limit that is set $50 \%$ of the time assuming the absence of a source. The discovery potential represents instead the flux required to have a $5 \sigma$ discovery, knowing this will be detected only $50 \%$ of the time. Curves have been produced assuming the best case scenario, that is when the source extension in the injector matches the extension of the likelihood scan, and considering an $E^{-2}$ power law. The Southern Sky performance is penalized by the higher background rates. 


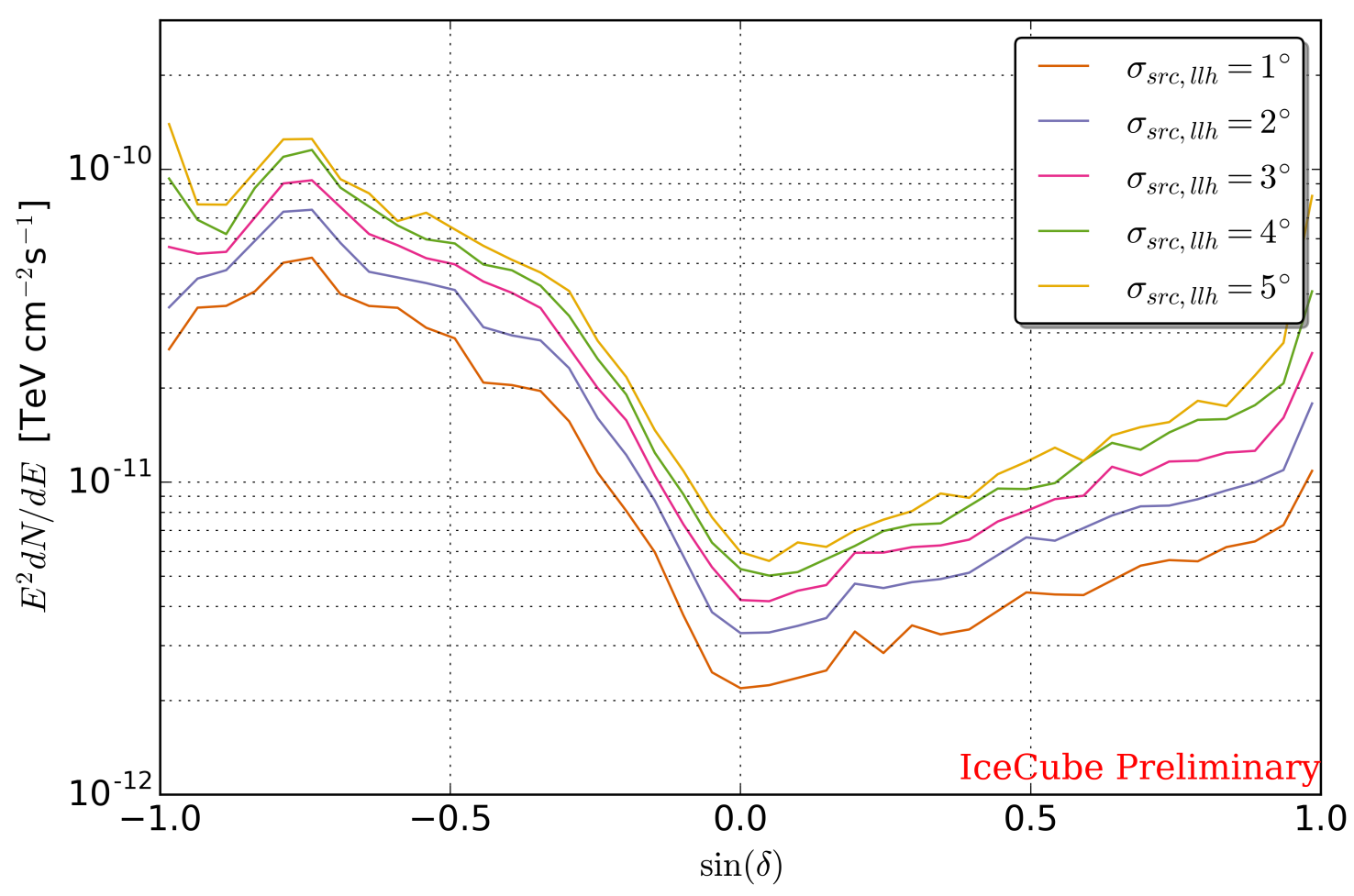

Figure 3: Discovery potential at 50\% confidence level for the five extensions considered and an $E^{-2}$ power law spectrum. The best case scenario is assumed, that is when the simulated source extension $\left(\sigma_{s r c}\right)$ matches exactly the extension of the likelihood scan $\left(\sigma_{l l h}\right)$.

\section{Results}

The aim of this analysis is to produce five significance skymaps. All-sky scans for source extensions of $1^{\circ}, 2^{\circ}, 3^{\circ}, 4^{\circ}$ and $5^{\circ}$ are shown in Figures $4 \mathrm{a}, 4 \mathrm{~b}, 4 \mathrm{c}, 4 \mathrm{~d}$ and $4 \mathrm{e}$, respectively. For each extension scan, the direction with the highest significance (marked with a black box on the skymaps) has been selected and the post-trial p-value calculated by comparing the result to the distribution of hotspots obtained from background-scrambled maps for each extension. Due to Earth's rotation, the probability density of the background is uniform in right ascension. It is therefore possible to produce background pseudo-experiment by using data that have kept the information of the declination but have been assigned a random value for the right ascension. Results of each scan are grouped in Table 1. All the results are consistent with the background-only hypothesis. The most significant among the maps is the $1^{\circ}$ extension with a post-trial $\mathrm{p}$-value of 0.1 . This result is however not significant, especially if considering a conservative trial factor of 5 to account for the fact that the maps are not independent. This is also the reason why hotspot locations for the $2^{\circ}$ and $3^{\circ}$ extensions and also for the $4^{\circ}$ and $5^{\circ}$ ones are the same. However, these locations have been checked and do not correspond to any know cataloged astrophysical object.

\section{Conclusions}

A search for extended sources has been performed using seven years of throughgoing muons. 


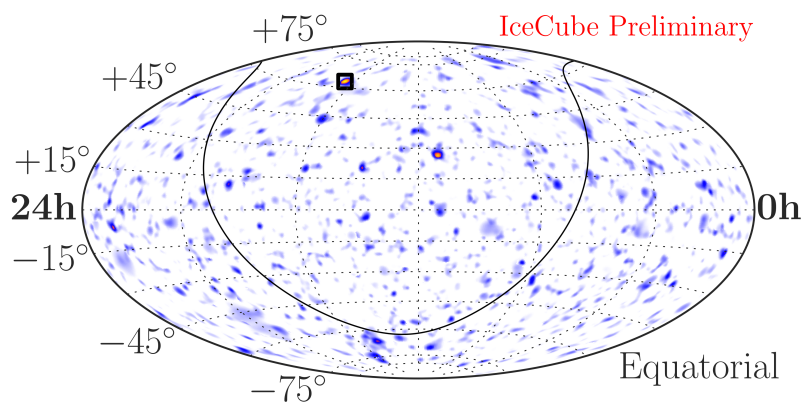

(a) $1^{\circ}$ source extension

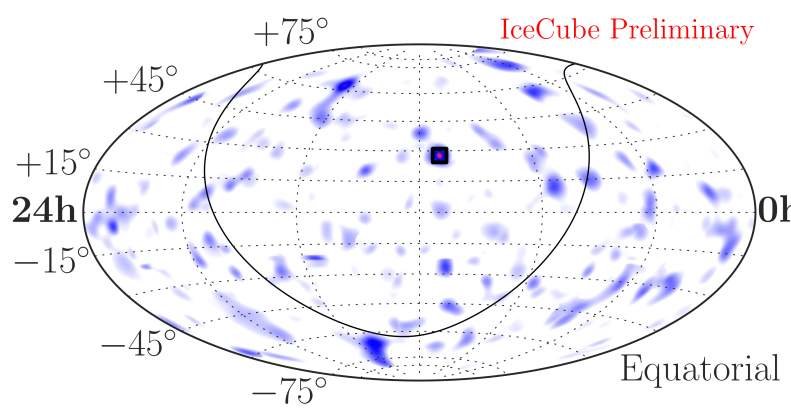

(c) $3^{\circ}$ source extension

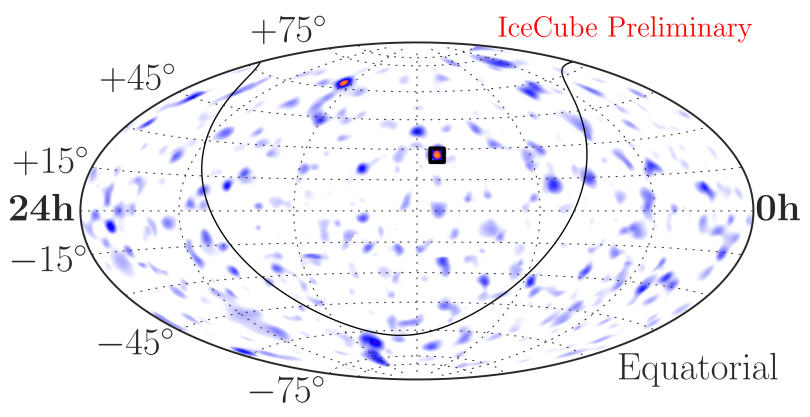

(b) $2^{\circ}$ source extension

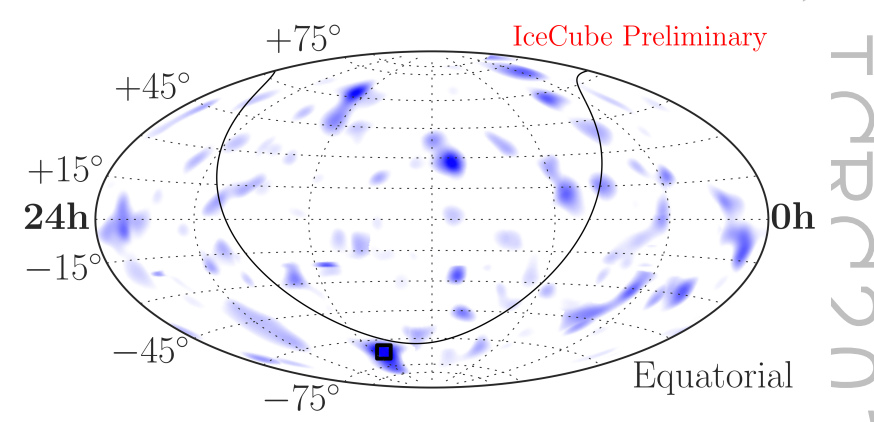

(d) $4^{\circ}$ source extension
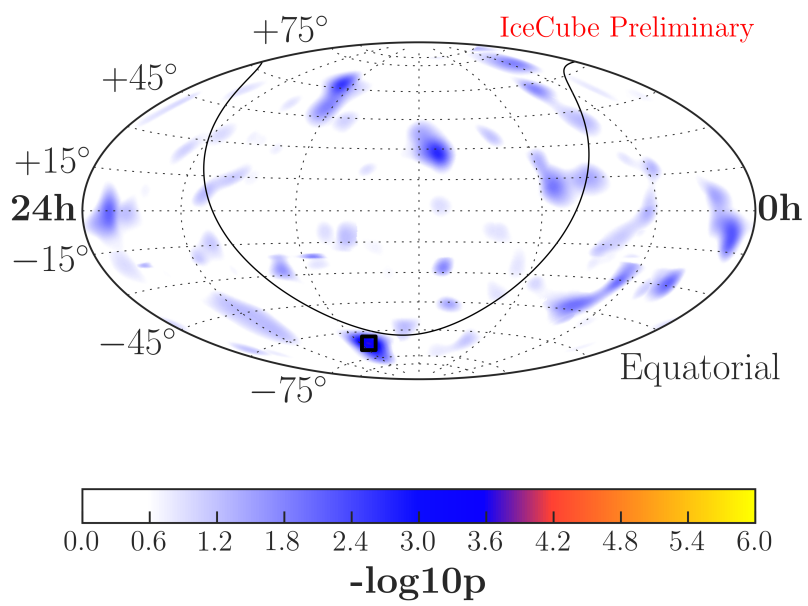

(e) $5^{\circ}$ source extension

Figure 4: Pre-trial significance skymaps for different source extension and seven years of throughgoing muons. The solid black line represents the galactic plane. The hottest spot is highlighted with a black box in each skymap. 


\begin{tabular}{ccccccc}
\hline Extension $\left[^{\circ}\right]$ & $\mathrm{RA}\left[^{\circ}\right]$ & $\mathrm{DEC}\left[^{\circ}\right]$ & $\hat{n}_{s}$ & $\hat{\gamma}$ & p-value (pre-trial) & p-value (post-trial) \\
\hline $1^{\circ}$ & 249.32 & 63.28 & 73.32 & 1.85 & $8.20 \times 10^{-7}$ & 0.10 \\
$2^{\circ}$ & 169.44 & 27.04 & 102.92 & 2.19 & $9.04 \times 10^{-6}$ & 0.30 \\
$3^{\circ}$ & 169.37 & 27.68 & 119.18 & 2.16 & $7.31 \times 10^{-5}$ & 0.58 \\
$4^{\circ}$ & 229.82 & -66.64 & 140.05 & 1.63 & $2.23 \times 10^{-4}$ & 0.78 \\
$5^{\circ}$ & 231.56 & -66.44 & 160.83 & 1.68 & $1.99 \times 10^{-4}$ & 0.60 \\
\hline
\end{tabular}

Table 1: Summary of the results from the extended all-sky survey. The coordinates of the most significant spots located for each source extension hypothesis are given together with the respective p-values. The post-trial p-values are all consistent with the background-only hypothesis.

Data have been taken with different detector configurations, from the 40-string to the final 86-string layout. Given the source extension is not known a priori, five extensions from $1^{\circ}$ to $5^{\circ}$ have been considered. A novel likelihood method has been implemented, that accounts and corrects for the signal contamination in the background obtained by scrambling technique. Five different significance skymaps have been produced, and a p-value has been calculated for the hottest spot of each map. All the five maps are consistent with the background-only hypothesis.

\section{References}

[1] IceCube Collaboration, M. G. Aartsen et al., JINST 12 (2017) P03012.

[2] IceCube Collaboration, PoS ( ICRC2015) 1081 (2016).

[3] IceCube Collaboration, M. G. Aartsen et al., ApJ 833 (2016) 3.

[4] IceCube Collaboration, M. G. Aartsen et al., ApJ 835 (2017) 151.

[5] IceCube Collaboration, M. G. Aartsen et al., ApJ 796 (2014) 109.

[6] H.E.S.S. Collaboration, F. Aharonian et al., A\&A 477 (2008) 353-363.

[7] Telescope Array Collaboration, R. Abbasi et al., ApJ 790 (2014) L21.

[8] KASCADE-Grande Collaboration, PoS ( ICRC2 015) 812 (2016).

[9] J. Braun et al., ApJ 29 (2008) 299-305.

[10] IceCube Collaboration, M. G. Aartsen et al., Phys. Rev. D 91 (2015) 122004. 